\title{
Thyroid Hemiagenesis: Role of an Oneologist
}

Pai VD, Nair D, Datta S, Chaturvedi P

Department of Surgical Oncology, Tata Memorial Centre, Mumbai, India.

\section{Abstract:}

Thyroid hemiagenesis is a rare congenital anomaly in which one lobe of thyroid fails to develop; which may or may not be associated with failure of development of the isthmus. Most of the patients remain asymptomatic. Thyroid pathologies are more common in these patients than the normal population. Euthyroid cases without any above mentioned disorders need reassurance for the patient. Importance of this case lies in two important things. First thyroid hemiagenesis is associated with increased incidence of other associated disorders including malignancy. Hence close follow up is essential. Secondly, when in euthyroid status, proper patient counselling and avoidance of unnecessary surgery are of paramount importance to avoid permanent hypothyroidism.

Key words: Thyroid Dysgenesis, Thyroid Neoplasms, Hypothyroidism, Thyroid Function Tests, Goiter.

\section{Introduction}

Thyroid hemiagenesis is a rare congenital anomaly in which one lobe of thyroid fails to develop; which may or may not be associated with failure of development of the isthmus. True incidence is difficult to estimate in view of the asymptomatic nature of the disease. With increasing use of imaging modalities, there has been an increase in the number of such cases detected quite frequently though its aetiology, associated pathology and its clinical implications in the day to day practice remain a topic of debate. Here we are presenting a teenage girl presenting with neck swelling who was found to have hemiagenesis of thyroid lobe.

\section{Case Report}

An eleven year old, otherwise healthy girl presented with history of swelling in front of the neck since 6 months. There were no symptoms suggestive of hypothyroidism or hyperthyroidism. Local examination revealed an enlargement of right lobe of thyroid [Fig.1a]. There were no neck nodes. Thyroid function test showed increased TSH level with normal T3 and T4. Ultrasound examination of neck revealed enlarged right lobe of thyroid with homogenous echogeneity and absence of any hypoechoeic areas [Fig. $1 \mathrm{~b}$ ]. Left lobe and isthmus were absent. No ectopic thyroid tissue was detected on scintigraphy [Fig. 1c]. She

Corresponding Author: Dr. Deepa Nair

Email: drdeepanair@hotmail.com

Received: January 29, 2015 | Accepted: April 24, 2015 | Published Online: April 30, 2015

This is an Open Access article distributed under the terms of the Creative Commons Attribution License (creativecommons.org/licenses/by/3.0)

Conflict of interest: None declared | Source of funding: Nil | DOl: http://dx.doi.org/10.17659/01.2015.0047 
was referred to our centre as fine needle aspiration cytology done from an outside centre revealed atypical cells. These slides were reviewed by our pathologist which revealed normal thyroid tissue. She was started on levothyroxine 100 micrograms OD considering her age and slightly elevated TSH. Patient has been followed up for more than a year. She is asymptomatic, off thyroxine and ultrasound neck is normal.

\section{Discussion}

Thyroid hemiagenesis (TH) is a rare congenital anomaly. The first case report was published way back in 1886 [1]. The exact prevalence is difficult to estimate since most remain asymptomatic. Population based studies have estimated the prevalence of $0.05-0.16 \%$ [2]. Review of literature revealed a systematic analysis of a series of 40 cases of thyroid agenesis [3].

Exact aetiology of TH is unknown, though both sporadic and familial cases have been reported. Advanced maternal age, trisomy 22 and FOXE 1 polyalanine tract length polymorphism have been found to be associated with thyroid hemiagenesis [4]. It is found more commonly in females with female: male ratio of $3: 1$. The probable reason for female predominance is likely to be higher number of thyroid pathologies in them with higher detection rate. It more commonly involves left lobe than right for unknown reasons with left: right ratio being 4:1 [5]. Associated absence of isthmus is observed more often when agenesis affected right lobe but not the left lobe [3]. Clinically these patients may be euthyroid, or hyperthyroid or hypothyroid with derangement of thyroid function seen in $38-47 \%$ of patients [6]. It has been observed that these patients have higher levels of TSH and FT3 compared to normal individuals. The probable reason being impaired thyroid reserve resulting in higher TSH level and increased peripheral conversion of T4

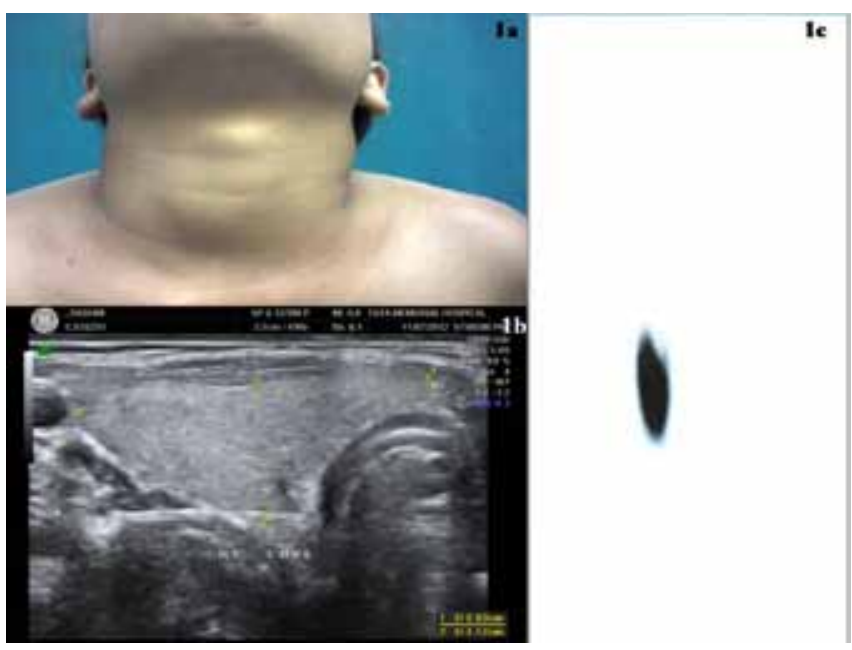

Fig.1:(a) Patient profile. (b) Ultrasound neck showing agenesis of left lobe. (c) Scintiagraphy showing agenesis of left lobe.

to T3 [7]. This also results in the hypertrophy of remaining lobe of thyroid as in our case. Higher TSH is also responsible for higher incidence of diffuse and nodular goitre in these patients [3].

Thyroid pathologies are more common in these patients than the normal population. Various pathologies reported are multinodular goitre, Grave's disease, Hashimoto's thyroiditis, papillary thyroid cancer and primary hyperparathyroidism $[8,9]$. Incidence of thyroid neoplasms is higher in these set of patients both as a result of higher TSH levels and due to higher incidence of nodules in them.

Ultrasound neck is the key diagnostic modality of choice for diagnosis of developmental disorders of the thyroid [10]. CT and MRI do not add any additional information to ultrasound and hence are not preferred. Scintigraphy is not routinely indicated though it is helpful in identifying the presence of ectopic thyroid tissue. Fine needle aspiration may be required when ultrasound shows suspicious features. 
Euthyroid cases without any above mentioned disorders need reassurance and regular follow up for the patient. Many of these individuals who are euthyroid and asymptomatic at presentation may become hypothyroid when basal requirement of thyroid hormones increase such as pregnancy or puberty. Hypothyroidism needs to be treated with levothyroxine in appropriate doses at the earliest [3].

\section{Conclusion}

Regular follow up of the patient is mandatory in patients of thyroid hemiagenesis as it is associated with increased incidence of associated disorders including malignancy. Proper patient counselling and avoidance of unnecessary surgery are of paramount importance to avoid permanent hypothyroidism in euthyroid patients.

\section{References}

1. Handfield-Jones. Primary hyperparathyroidism with thyroid hemiagenesis. In: Handbuch der Systematischen Anatomie des Menschen. Henle J, Ed. Sohn: Friedrich Vlewig und Braunschweig, 1866:538 (in German).

2. Korpal-Szczyrska M, Kosiak W, Swieton D. Prevalence of thyroid hemiagenesis in an asymptomatic schoolchildren population. Thyroid. 2008; 18(6):637-639.

3. Ruchala $M$, Szczepanek E, Szaflarski W, Moczko J, Czarnywojtek A, Pietz L, et al. Increased risk of thyroid pathology in patients with thyroid hemiagenesis: results of a large cohort case-control study. Eur J Endocrinol. 2010;162(1):153-160.

4. Szczepanek E, Ruchala M, Szaflarski W, Budny B, Kilinska $L$, Jaroniec $M$, et al. FOXE1 polyalanine tract length polymorphism in patients with thyroid hemiagenesis and subjects with normal thyroid. Horm Res Paediatr. 2011 ;75(5):329334.

5. Melnick JC, Stemkowski PE. Thyroid hemiagenesis (hockey stick sign): a review of the world literature and a report of four cases. J Clin Endocrinol Metab. 1981;52(2):247-251.

6. Maiorana R, Carta A, Floriddia G, Leonardi D, Buscema M, Sava L, et al. Thyroid hemiagenesis: prevalence in normal children and effect on thyroid function. J Clin Endocrinol Metab. 2003;88(4): 1534-1536.

7. Bhartiya S, Verma A, Basu S, Shukla V. Congenital thyroid hemiagenesis with multinodular goiter. Acta Radiol Short Rep. 2014;3(9):2047981614530286.

8. Baldini M, Orsatti A, Cantalamessa L. A singular case of Graves' disease in congenital thyroid hemiagenesis. Horm Res. 2005;63(3):107-1 10.

9. Wang J, Gao L, Song C. Thyroid hemiagenesis associated with medullary or papillary carcinoma: report of cases. Head Neck. 2014;36(1 1):E106-111.

10. Ayaz ÜY, Ayaz S, Döğen ME, Api A. Ultrasonographic and scintigraphic findings of thyroid hemiagenesis in a child: report of a rare male case. Case Rep Radiol. 2015;2015:917504. 